\title{
APR Reduction Technique based on OFDM Pilot Filter Bank Limiting for Satellite Mobile Communications
}

\author{
Tianfang Dai ${ }^{1, a}$ \\ ${ }^{1}$ School of Mechanical and Electrical Engineering, University of Electronic Science and Technology of China, 611731 Chengdu, China
}

\begin{abstract}
Combined with OFDM (Orthogonal Frequency Division Multiplexing), satellite mobile communications will effectively achieve on-demand communication in areas with an ultra-low density of users. With OFDM multiple access optimization, the bandwidth utilization efficiency can be increased by 5 to 10 times. However, satellites are power-constrained systems, so higher PAPR requires greater power backoff, resulting in a decline in satellite transmission capacity. To use OFDM technology in satellites, there are problems such as reduced transmission capacity resulted from high PAPR, complication of lowering PAPR, and difficulty in hardware implementation. In order to deal with the problem of high bit error rate and hardware implementation difficulties in PAPR reduction technique of non-orthogonal frequency division multiplexing, this paper proposes a limiting PAPR reduction technique with OFDM pilot filter banks for satellite mobile communications. Firstly, the applicability of OFDM in satellite mobile communications is analyzed, and the influence of high PAPR on satellite power utilization and the influence of frequency shift sensitivity on inter-satellite communication interference are obtained. Then design the PAPR reduction technique based on the pilot filter bank. By setting the tunable filter bank to the pilot, the sideband power suppression in the OFDM frequency domain is realized, and the PAPR of the OFDM signal is reduced. Finally, the experimental results show that the PAPR performance is improved by $3 \mathrm{~dB}$ without reducing the bit error rate.
\end{abstract}

\section{Introduction}

Satellite mobile communication with OFDM is a highly efficient method for human beings to connect with each other at any time, in any space and with arbitrary quality by further utilizing electromagnetic spectrum and transmission power. It will effectively realize on-demand communications in areas with an ultra-low user density such as oceans, mountains and deserts.

After the introduction of OFDM, satellite mobile communications can effectively address the bandwidth shortage and frequency selective fading. In addition, this will effectively promote the development of key technologies such as high energy efficiency modulation coding, dynamic channel estimation, dynamic antiinterference and multipath mitigation technology.

Research into the use of OFDM technology in satellite mobile communications has attracted the attention of powerful countries across the world. US ONEWEB has invested nearly 10 billion US dollars to plan thousands of small satellites. By adopting OFDM-based communication system, it offers $50 \mathrm{Mbps}$ broadband communication capability, and will provide affordable network connection in remote areas or areas with backward Internet infrastructure construction. The European Union has built a DVB-T communication system for digital video broadcasting. It has designed a multiple access multiplexing method for uplink OFDM, which effectively improves the throughput of broadband broadcasting satellites and serves live video broadcasting and backbone network traffic unloading around the world. The 3GPP organization has made satellites an important access component for future networks in $\mathrm{B} 4 \mathrm{G}$ and $5 \mathrm{G}$, forming an important option the OFDM as a satellite communications system for non-terrestrial networks (NTN). [1]

With OFDM multiple access optimization, bandwidth utilization efficiency can be increased by 5 to 10 times, which will make high-throughput services possible, greatly boosting the development of high-definition videos and virtual reality services and creating trillionscale traffic as well as new businesses. Meanwhile, after the high PAPR is addressed, the use of OFDM in satellites can greatly increase the communication capability, thus achieving more new "satellite plus" services and facilitating the formation of a new satellite industry chain.

Currently, PAPR reduction techniques of OFDM in satellites can be classified into limiting, coding, and scrambling. [2] The limiting method is to limit the signal amplitude to the nonlinear threshold of the amplifier, which will also cause nonlinear distortion while reducing the PAPR, bringing more serious in-band noise and outof-band interference, and finally increasing the bit error rate. The coding method finds the combination of code 
elements corresponding to the lowest PAPR by traversing the power amplitude value obtained through mapping different code combinations. However, the calculation involved in this method is highly complicated and the PAPR is difficult to be reduced after the subcarriers are increased. The scrambling method is to conduct a weighting algorithm on the signal with a special scrambling sequence, and then select an OFDM symbol with a small PAPR value for transmission, which requires multiple FFT/IFFT calculations and consumes more pilot symbols.

To sum up, there are two difficulties facing PAPR reduction in satellites [3]:

First, it's difficult to control the bit error rate of transmission when reducing the PAPR.

Second, hardware implementation is challenging because the method of PAPR reduction is complicated.

In order to deal with the problem of high bit error rate and hardware implementation difficulties in PAPR reduction technique non-orthogonal frequency division multiplexing, this paper proposes a limiting PAPR reduction technique with OFDM pilot filter banks for satellite mobile communications. Firstly, the applicability of OFDM in satellite mobile communications is analyzed, and the influence of high PAPR on satellite power utilization and the influence of frequency shift sensitivity on inter-satellite communication interference are obtained. Then design the PAPR reduction technique based on the pilot filter bank. By setting the tunable filter bank to the pilot, the sideband power suppression in the OFDM frequency domain is realized, and the PAPR of the OFDM signal is reduced. Finally, the experimental results show that the PAPPR performance is improved by $3 \mathrm{~dB}$ without reducing the bit error rate with the method of this paper.

\section{Applicability Analysis of OFDM in Satellite Mobile Communications}

\subsection{Fundamentals of OFDM}

As a multiple access method for LTE and 5G mobile communication technologies, OFDM has the advantage of high spectrum utilization efficiency. The smallest unit that constitutes an OFDM baseband signal is an OFDM symbol, and one OFDM symbol includes numerous synthesis signals of modulated subcarriers, as shown in Figure 1.

A continuous OFDM signal can be expressed as [4]

$$
x(t)=\frac{1}{\sqrt{N}} \sum_{k=}^{N-1} X_{k} e^{j 2 k \pi f_{k} t}, 0 \leqslant t \leqslant T
$$

Here, $T$ is the symbol period of OFDM, $N$ is the number of subcarriers, $X=\left\{X_{k}, k=0, \ldots N-1\right\} \quad$ is symbols of frequency domain, $X_{k}$ represents the modulated data of the subcarrier $k$, and $f_{k}$ is the carrier frequency of the subcarrier $k, f_{k}=k / T$.

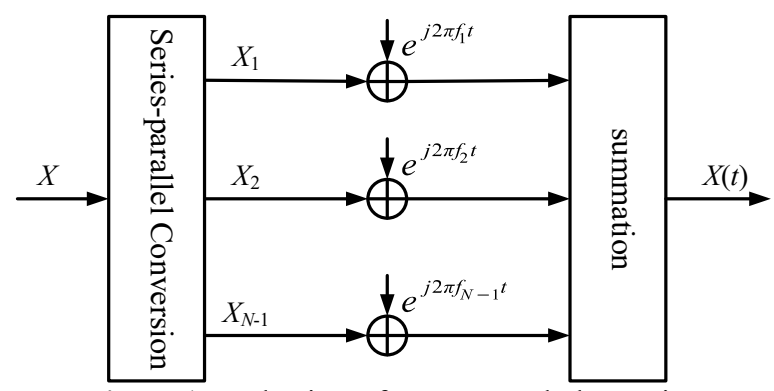

Figure 1 Mechanism of OFDM symbol mapping

The equation presented above demonstrates the continuous OFDM signal. After Nyquist sampling, a discrete OFDM signal can be obtained.

$$
x_{n}=\frac{1}{\sqrt{N}} \sum_{k=0}^{N-1} X_{k} e^{j 2 k \pi n / N}=\sqrt{N} \cdot I D F T_{N}\left\{X_{k}\right\}, 0 \leqslant n \leqslant N
$$

Here, IDFTN represents the N-order discrete Fourier transform. Equation (2) shows that OFDM can be modulated with fast Fourier transform (FFT),

$$
\left\langle e^{j m t}, e^{j n t}\right\rangle=\int_{-\infty}^{\infty} e^{j(m-n) t} d t=\delta(m-n)
$$

That is, the inner product between subcarriers of different frequencies is 0 , indicating that the subcarriers are orthogonal to each other (as shown in Figure 2).

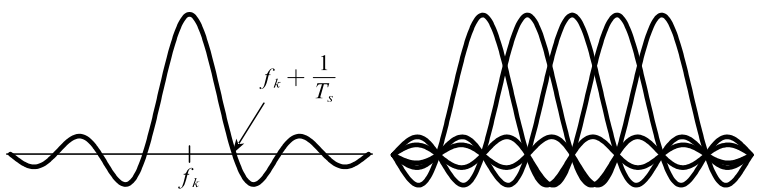

Figure 2 Spectral overlay of OFDM subcarriers

As we can see in Figure 2, compared with the conventional frequency division multiplexing (FDM), the subcarriers of the OFDM overlap each other in the frequency domain, and the guard bands are not needed to separate adjacent carriers. Since the orthogonality prevents other subcarriers from affecting the sampling and valuing of the current carrier, the information carried by the carrier group can be completely demodulated at the receiving end. In addition, since the guard band is not required and the subcarriers can overlap each other, OFDM has high spectral efficiency and can transmit more bit information in a limited band [6]. Therefore, if used as the multiple access method, OFDM can improve the spectrum utilization efficiency of satellites.

\subsection{Analysis of the Impact of High PAPR on Satellite Power Utilization}

The OFDM signal is a superposition of multiple subcarriers. If multiple carriers are stacked in phase at a certain time, the instantaneous power of the superposed signal will be much higher than the average power, resulting in a very high peak.

The PAPR of an OFDM signal is defined as the ratio of the peak power of the signal to the average power within a single OFDM symbol. The PAPR of the continuous time signal $x(t)$ is defined as: 


$$
\begin{aligned}
& \operatorname{PAPR}[x(t)]=\frac{\max _{0 \leqslant t \leqslant T}\left[|x(t)|^{2}\right]}{P_{a v}} \\
& \operatorname{PAPR}\left(x_{n}\right)=10 \lg \frac{\max \left\{\left|x_{n}\right|^{2}\right\}}{E\left\{\left|x_{n}\right|^{2}\right\}}
\end{aligned}
$$

Generally, the complementary cumulative probability distribution function (CCDF) of the PAPR is used to measure its distribution, which indicates the probability that the PAPR exceeds a given value $\lambda[7]$.

$$
P(P A P R>\lambda)=1-\left(1-e^{-\lambda}\right)^{N}
$$

In an OFDM system, there are some nonlinear response devices in the transmitter and receiver, such as $\mathrm{A} / \mathrm{D}$ and $\mathrm{D} / \mathrm{A}$ converters, mixers and power amplifiers (PA), which may cause nonlinear distortion [8]. Usually, the distortion caused by HPA mainly demonstrates in two forms of phase offset and amplitude distortion, which are usually represented by $\mathrm{AM} / \mathrm{AM}$ (amplitude distortion of input signal) and AM/PM (phase distortion of input signal). If the input OFDM signal is expressed as

$$
x=|x| e^{j a r g(x)}=r e^{j \phi}
$$

The output of the nonlinear system is as follows:

$$
g(x)=A(r) e^{j(\phi+\Phi(r))}
$$

Here, $A(r)$ and $\Phi(r)$ represent the AM/AM and $\mathrm{AM} / \mathrm{PM}$ conversions of the signal. At present, the traveling wave tube amplifier (TWTA) is used more on satellites. As for the TWTA amplifier, the AM/AM and $\mathrm{AM} / \mathrm{PM}$ conversions can be expressed as

$$
\begin{aligned}
& A(r)=\frac{\alpha r}{1+\beta r^{2}} \\
& \Phi(r)=\frac{\gamma r^{2}}{1+\varepsilon r^{2}}
\end{aligned}
$$

Where $r$ is the input amplitude of TWTA, $A(r)$ and $\Phi(r)$ represent the amplitude response and phase response of TWTA, $\alpha, \beta, \gamma, \varepsilon$ are four adjustable parameters. The curve of $A(r)$ and $\Phi(r)$ of TWTA is shown in Figure 3. The parameters used in the figure are $\alpha=1.9638, \beta=0.9945, \gamma$ $=2.5293, \varepsilon=2.8168$.

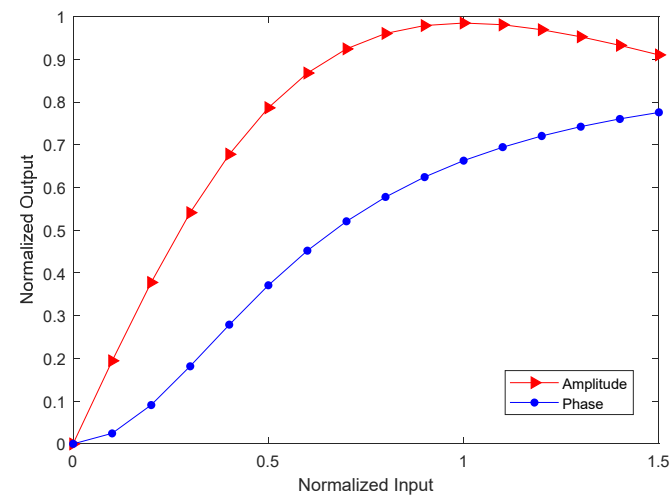

Figure 3 Nonlinear saturation of satellite TWTA

As shown in Figure 3, the power amplification TWTA is non-linear. Above the supersaturation point, as the input power increases, the output power increases by a small amount. If the PAPR is not reduced, in order to reduce nonlinear distortion caused by TWTA in satellites, it is necessary to set the operating point below the saturation point, which requires a large power back-off. However, the power of the satellite is limited, meaning the required power back-off is hard to meet. Therefore, reducing PAPR is the best solution.

\subsection{Analysis of the Impact of Frequency Shift Sensitivity on Inter-symbol Interference in Satellite Communications}

The LEO satellite moves at a high speed relative to the ground, so there is a significant Doppler Effect.

The relative Doppler shift $\Delta f$ is

$$
\Delta f=\frac{v}{c} f
$$

Where $v$ is the velocity component of the satellite motion in the direction of the electromagnetic wave propagation, namely, the radial velocity; $c$ is the light speed; $f$ is the frequency of the transmitted signal. Therefore, $\Delta f$ exhibits an S-type variation law as $v$ changes.

The Doppler Effect in the satellite-to-ground data transmission system is much more serious than that in the ground wireless communication system. Also, the local dynamic law appears, and the maximum rate of change can hit several kHz/s.

The local oscillator frequency at the receiving end is $f_{O}$. it is held that the local oscillator deviation is much smaller than the sampling frequency $N / T_{s}$. After the receiver is down-converted, the signal is sampled, namely, $\mathrm{t}=k T_{s} / N$. The following equation can be obtained.

$$
r_{k}=e^{j 2 \pi\left[f_{c}\left(1+\beta_{k}\right)-f_{O}\right] k T_{s} / N} \cdot s_{k}+\omega_{k}
$$

Where,

$$
\beta_{k}=\beta_{k T_{s} / N}, s_{k}=\frac{1}{\sqrt{N}} \sum_{n=0}^{N-1} a_{n} e^{j 2 \pi \cdot\left(1+\beta_{t}\right) \cdot n}
$$

If the normalized frequency offset is defined as $f_{\delta}$, the following equation can be obtained.

$$
f_{\delta} \triangleq \frac{f_{c}\left(1+\beta_{t}\right)-f_{o}}{f_{s}}=\left(f_{c}\left(1+\beta_{t}\right)-f_{o}\right) T_{s}
$$

The normalized frequency offset represents the ratio of the frequency offset to the carrier spacing. In order to focus on the frequency offset effect caused by the Doppler Effect, it is temporarily assumed that, in the above system, the local oscillator has a small deviation, which is negligible compared to the Doppler frequency offset, namely, $f_{O} \approx f_{c}$. At the same time, $f_{\delta} \approx f_{c} \beta k T_{s}$.

After applying FFT to conduct OFDM modulation on signal $r_{k}$, we can derive the demodulation sequence $\left\{z_{m} \mid\right.$ $0 \leq m \leq N-1\}$, namely,

$$
\begin{aligned}
z_{m} & =\frac{1}{\sqrt{N}} \sum_{k=0}^{N-1} r_{k} e^{-j 2 \pi m k / N} \\
& =\frac{1}{N} \sum_{n=0}^{N-1} a_{n} \sum_{k=0}^{N-1} \exp \left(\frac{j 2 \pi k\left(f_{\delta}+\beta n+n-m\right)}{N}\right) \\
& =c_{m} a_{m}+\sum_{\substack{n=0 \\
n \neq m}}^{N-1} c_{n} a_{n}
\end{aligned}
$$

Where $c_{m} a_{m}$ is the symbol on the demodulated subcarrier $m m ; c_{m}$ is the loss factor caused by Doppler; $c_{n} a_{n}$ 
and $n \neq m$ represent the ICI resulted from the Doppler effect, and $c_{n}$ is defined as the interference intensity factor.

$$
\begin{aligned}
& c_{m}=\frac{1}{N} \sum_{k=0}^{N-1} \exp \left(\frac{j 2 \pi k\left(f_{\delta}+\beta n\right)}{N}\right) \\
& c_{n}=\frac{1}{N} \sum_{k=0}^{N-1} \exp \left(\frac{j 2 \pi k\left(f_{\delta}+\beta n+n-m\right)}{N}\right), n \neq
\end{aligned}
$$

It can be seen that the carrier frequency offset destroys the orthogonality between the subcarriers, thereby introducing inter-carrier interference. [9]

\section{PAPR Reduction Technology for Pilot Filter Bank}

At present, the methods for reducing the PAPR of satellite OFDM mainly include limiting, coding and scrambling. Figure 4 and Table 1 illustrate the performance comparison results of different methods for reducing PAPR under the same conditions.

As can be seen from Figure 4 and Table 1, all of these schemes have an adverse effect on bit error and transmission performance while reducing PAPR the signal. As for the distortion schemes, PAPR reduction effect is evident despite its simplicity, but its nonlinear processing also causes a significant reduction in system performance. As for non-distortion schemes, although they do not affect the bit error rate of the signal, they tend to reduce the data transmission rate and increase the computational complexity. Therefore, we use the filter bank to correct the energy band of the pilot to improve its performance.

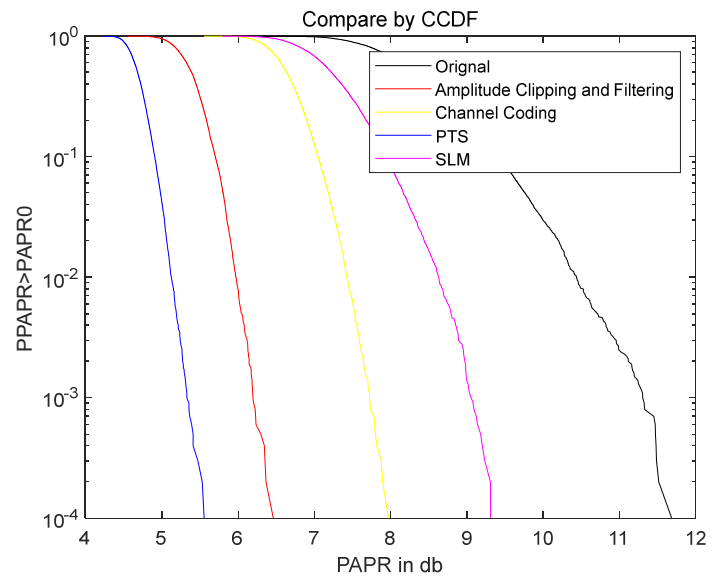

Figure 4 Performance of different methods for PAPR reduction

Table 1 Summary of performance of different methods for PAPR reduction

\begin{tabular}{|l|l|l|l|l|l|}
\hline $\begin{array}{l}\text { Metho } \\
\mathrm{d}\end{array}$ & $\begin{array}{l}\text { Distortio } \\
\mathrm{n}\end{array}$ & $\begin{array}{l}\text { Transmit } \\
\text { Power }\end{array}$ & $\begin{array}{l}\text { Data } \\
\text { quantity }\end{array}$ & $\begin{array}{l}\text { Sending-end } \\
\text { processing }\end{array}$ & $\begin{array}{l}\text { Receiving-end } \\
\text { processing }\end{array}$ \\
\hline $\begin{array}{l}\text { Limiti } \\
\mathrm{ng}\end{array}$ & Yes & Decrease & No change & $\begin{array}{l}\text { Limiting } \\
\text { amplitude } \\
\text { wave filter }\end{array}$ & None \\
\hline $\begin{array}{l}\text { Codin } \\
\mathrm{g}\end{array}$ & No & $\begin{array}{l}\text { No } \\
\text { change }\end{array}$ & Decrease & $\begin{array}{l}\text { Coding or } \\
\text { checking table }\end{array}$ & $\begin{array}{l}\text { Coding or } \\
\text { checking table }\end{array}$ \\
\hline PTS & No & $\begin{array}{l}\text { No } \\
\text { change }\end{array}$ & Decrease & $\begin{array}{l}\text { M IFFT,WM- } \\
\text { Sum of } \\
\text { Composite } \\
\text { Vectors }\end{array}$ & $\begin{array}{l}\text { Extracting } \\
\text { sideband } \\
\text { information, } \\
\text { inverse PTS } \\
\text { process }\end{array}$ \\
\hline SLM & No & $\begin{array}{l}\text { No } \\
\text { change }\end{array}$ & Decrease & $\begin{array}{l}\text { M IFFT } \\
\text { IFtracting } \\
\text { sideband } \\
\text { information, } \\
\text { inverse PTS } \\
\text { process }\end{array}$ \\
\hline
\end{tabular}

The method mainly uses OQAM modulation to generate a sequence in which the real part and the imaginary part alternate, filters through the pilot filter, removes the filter length delay by half, and finally performs inverse Fourier transform to obtain the final OFDM signal. The key to this method is the design of the pilot filter, which first filters modulated signals in each subcarrier. The filter is characterized by an overlap factor $K$, which is the number of overlapping multi-carrier symbols in the time domain. The pilot filter order can be chosen to be $2 \times K-1$, where $K=2,3$ or 4 . An IFFT with a length of $N \times K$ is used, where the sign overlaps with the delay of $N / 2$, and $N$ is the number of subcarriers.

In order to achieve full capacity, offset quadrature amplitude modulation (OQAM) processing is employed. The real and imaginary parts of the complex data symbol are not transmitted at the same time because the imaginary part is delayed by half of the symbol duration. The transmit-end processing is shown in Figure 5.

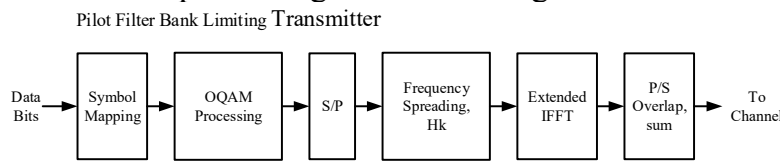

Figure 5 Block diagram of multi-filter group modulation implementation

Through simulation, the power spectral density of the PAPR reduction technology in pilot filter bank is shown in Figure 6.

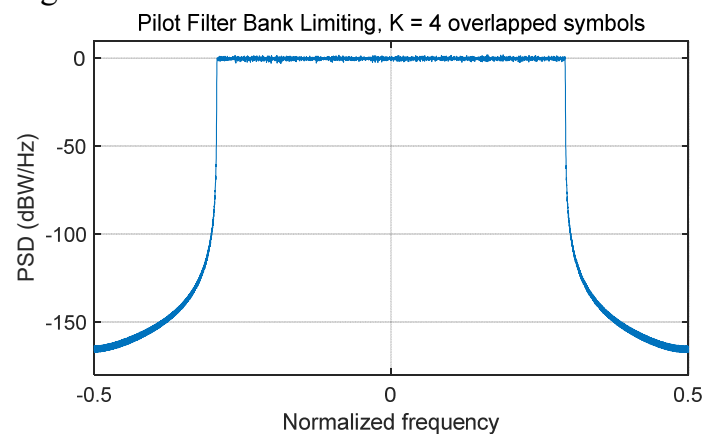

Figure 6 The spectral density of multi-filter group modulation

It can be seen that the in-band power is limited to OdBW after the new OFDM beam is formed. However, the out-of-band rejection can be below negative $150 \mathrm{dBW}$, with a very low out-of-band leakage, which can prevent high PAPR value.

As a comparison, the conventional OFDM power spectrum is as follows:

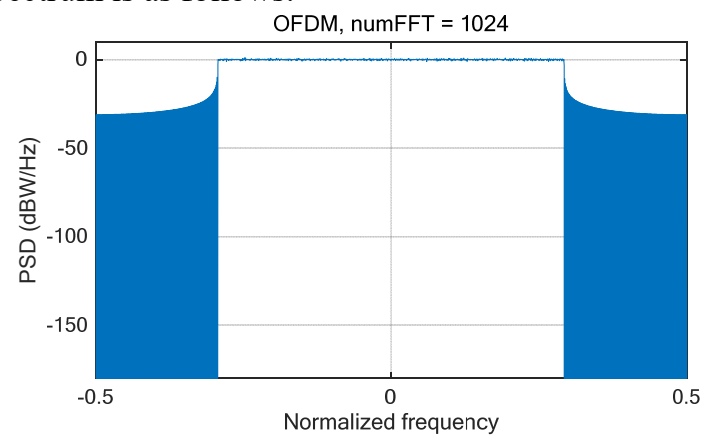

Figure 7 The spectral density of OFDM

Comparing the spectrogram of the pilot filter bank with that of the conventional OFDM, it can be seen that the 
feature of the out-of-band leakage of the conventional OFDM is greatly improved in the pilot filter bank system.

To analyze the anti-noise performance of the pilot filter bank, a demodulator including matched filtering is constructed, followed by OQAM separation to form the data symbols received. They are de-mapped into bits and the resulting bit error rate is determined.

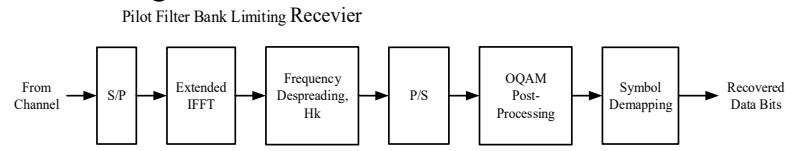

Figure 8 Block diagram of multi-filter group demodulation implementation

The simulation results show that when the overlap factor $K$ is 4 and the SNR is $12 \mathrm{~dB}$, the bit error rate is zero.

\section{Conclusion}

This paper designs the PAPR reduction technique based on the pilot filter bank. Combined with OQAM modulation, it decreases the out-of-band leakage of the OFDM system, which reduces the PAPR of the OFDM signal. The simulation results show that the bit error rate is zero when the SNR is $12 \mathrm{~dB}$. With a lower PAPR, The new multi-filter bank technology can fully utilize the limited power of satellites while ensuring a lower bit error rate of communication and reducing complexity for coding and decoding.

\section{References}

1. Qing-hua TIAN,Qi ZHANG,Ying TAO,Nai-jin LIU,Xiang-jun XIN,Li-jia ZHANG,Da-xiong XU. Performance analysis of broadband satellite communication system based on OFDM/TDM[J]. The Journal of China Universities of Posts and Telecommunications, 2013,20(3).

2. Sandeep Bhad, Pankaj Gulhane,A.S. Hiwale. PAPR Reduction Scheme for OFDM[J]. Procedia Technology,2012,4.

3. Parthasarathy Elavarasan, G. Nagarajan. A Summarization on PAPR Techniques for OFDM Systems[J]. Journal of The Institution of Engineers (India): Series B, 2015, Vol.96 (4), pp.381-389

4. Hyunseuk Yoo,Frédéric Guilloud,Ramesh Pyndiah. PAPR reduction for LDPC coded OFDM systems using binary masks and optimal LLR estimation[J]. Signal Processing,2011,91(11).

5. Arun Kumar,Shikha Bharti. Design and Performance Analysis of OFDM and FBMC Modulation Techniques[J]. The Scientific Bulletin of Electrical Engineering Faculty,2017,17(2).

6. Leonid Uryvsky,Serhii Osypchuk. OFDM Signal Research with Varied Subcarriers Number[J]. Transport and Telecommunication Journal,2016,17(3).

7. Tanmoy Saha,Shreyasi Chakrabarty,Subhankar Bhattacharjee,Sanjib Sil. Algorithm Based New Tone Reservation Method for Mitigating PAPR in OFDM
Systems[J]. International Journal of Electronics and Telecommunications,2017,63(3).

8. Chusit Pradabpet,Kobchai Dejhan. A new PAPR reduction in OFDM systems using PTS combined with APPR for TWTA nonlinear HPA[J]. Songklanakarin Journal of Science and Technology,2008,30(3).

9. Samarendra Nath Sur,Rabindranath Bera. Doppler Shift Impact On The MIMO OFDM System In Vehicular Channel Condition[J]. International Journal of Information Technology and Computer Science(IJITCS),2012,4(8). 